\title{
Graph Cospectrality using Neighborhood Matrices
}

\author{
A. D. Bessa I. C. Rocha-Neto \\ Instituto de Matemática \\ Universidade Federal da Bahia \\ Salvador-BA, Brazil \\ \{alineduartebessa, ivan.cr.neto\}@gmail.com
S. T. R. Pinho R. F. S. Andrade
Instituto de Física
Universidade Federal da Bahia
Salvador-BA, Brazil
\{suani, randrade\}@ufba.br
T. C. Petit Lobao *
Instituto de Matemática
Universidade Federal da Bahia
Salvador-BA, Brazil
thierry@ufba.br

Submitted: Jan 20, 2012; Accepted: Aug 14, 2012; Published: Aug 23, 2012

Mathematics Subject Classifications: 05C30, 05C50, 05C60

\begin{abstract}
In this note we address the problem of graph isomorphism by means of eigenvalue spectra of different matrix representations: the neighborhood matrix $\hat{M}$, its corresponding signless Laplacian $Q_{\hat{M}}$, and the set of higher order adjacency matrices $M_{\ell}$ s. We find that, in relation to graphs with at most 10 vertices, $Q_{\hat{M}}$ leads to better results than the signless Laplacian $Q$; besides, when combined with $\hat{M}$, it even surpasses the Godsil and McKay switching method.
\end{abstract}

Keywords: graph theory; cospectrality; neighborhood

\section{Introduction and Basic Definitions}

The absence of cospectral mates is relevant in identifying non-isomorphic graphs [5]. The adjacency matrix $A$ provides a large fraction of cospectral graphs in comparison to the Laplacian, $L$, and signless Laplacian , $Q[8,10,6]$. Haemers and Spence [8] have published a study enumerating the cospectral graphs (up to 11 vertices) in relation to

${ }^{*}$ Corresponding Author: Instituto de Matemática, Universidade Federal da Bahia Campus Universitário de Ondina, 40170-110 Salvador-BA, Brazil 
these matrices, later updated by Brouwer and Spence [3]. In this work, we systematically resume the issue by considering the spectra of matrices that emerge from neighborhood properties of graphs $[1,2]$, namely, $M_{\ell} \mathrm{s}, \hat{M}$, and $Q_{\hat{M}}$. Besides, since the combined use of different matrices spectra reduces the cospectrality incidence among graphs [10], we investigate the combinations $\hat{M} \& Q, \hat{M} \& Q_{\hat{M}}$ and $Q \& Q_{\hat{M}}$. This study suggests that these matrices and their combinations are relevant in identifying non-isomorphic graphs.

The higher order matrices $M_{\ell}$ and the neighborhood matrix $\hat{M}$ emphasize the neighborhood graph structure; the matrix $Q_{\hat{M}}$ has the analogous meaning as $Q$ for the matrix $A$. The neighborhood matrix $\hat{M}$ is a slight variation of the distance matrix [4], while the higher order matrices $M_{\ell}$ s stand for the adjacency matrices of each order of neighborhood. The following definitions, on simple graphs of diameter $d$, clarify these notions.

Definition 1. Two vertices $i$ and $j$ are said adjacent of order $\ell \in\{0,1, \ldots, d\}$ if and only if the shortest path between them has length $\ell$. This is denoted by $(i, j) \in O(\ell)$. If the vertices are not connected, then they are not adjacent in any order.

Definition 2. The higher order matrix of order $\ell$, denoted by $M_{\ell}$, is given as:

$$
\left(M_{\ell}\right)_{i, j}= \begin{cases}1, & \text { if }(i, j) \in O(\ell) \\ 0, & \text { otherwise }\end{cases}
$$

Two graphs are cospectral with respect to $M_{\ell}$ if and only if they have equal $M_{\ell}$ spectra for all $\ell$. It is possible to evaluate all $M_{\ell}$ s matrices by finite induction, which proceeds with polynomial time depending on the order of the graph, by

$$
M_{\ell}=\left(\bigoplus_{j=0}^{\ell-1} M_{j}\right) \otimes M_{1}-\left(\bigoplus_{j=0}^{\ell-1} M_{j}\right)
$$

in which the symbols $\oplus$ and $\otimes$ stand for addition and multiplication matrix operations, respectively, straightforward defined on their entries by the Boolean operations of addition and multiplication on the algebra $\{0,1\}$.

Definition 3. The neighborhood matrix $\hat{M}$ is given by $\hat{M}=\sum_{\ell=0}^{D} \ell M_{\ell}$.

Definition 4. Analogously to the classical notions of the Laplacian matrices, we define the signless Laplacian neighborhood matrix as $Q_{\hat{M}}=D_{\hat{M}}+\hat{M}$, with $\left(D_{\hat{M}}\right)_{i j}=\sum_{j=1}^{n} \hat{M}_{i j}$.

\section{Results}

To study the spectra we used as input the graph sets provided by Brendan McKay [9]. The eigenvalues were numerically calculated with double precision variables. For some matrices, cospectrality was also detected by the comparison among the integer coefficients of the characteristic polynomial. In spite of the higher computational cost, it increases the reliability of the comparison.

The neighborhood matrices present different properties regarding the classical ones. For instance, for the adjacency matrix $A$ and the Laplacian matrix $L$, if their associated 
graphs are cospectral, then they have the same number of edges; this property reduces the number of spectra that have to be determined in order to enumerate cospectral graphs. We have found that this property does not stand for $\hat{M}$; therefore our analysis requires more CPU time than the cospectrality analysis of $A$ and $L$. This issue, allied to the fact that we compare spectra by characteristic polynomials, explains the reason why our analysis proceeds at a slow pace and it is very hard to go further than 10 vertices.

Tables 1 and 2 present our results (boldface type) for the number and fraction of nonisomorphic graphs with cospectral mates according to the neighborhood matrices; we also include the results reported in [8] and [3].

Table 1: Number of non-isomorphic graphs with cospectral mates, until $n=10$, w.r.t. to $A, A \& \neg A, L, Q, M_{\ell} s, \hat{M}$ and $Q_{\hat{M}}$. The symbols $*$ and ${ }^{* *}$ refer to the values obtained in [3] and here, respectively, both of them replacing the value in [8].

\begin{tabular}{|l|l|l|l|l|l||l|l|l|}
\hline \hline$n$ & \# graphs & $A$ & $A \& \neg A$ & $L$ & $Q$ & $\mathbf{M}_{\ell} \mathrm{s}$ & $\hat{\mathbf{M}}$ & $Q_{\hat{\mathbf{M}}}$ \\
\hline 2 & 2 & 0 & 0 & 0 & 0 & $\mathbf{0}$ & $\mathbf{0}$ & $\mathbf{0}$ \\
3 & 4 & 0 & 0 & 0 & 0 & $\mathbf{0}$ & $\mathbf{0}$ & $\mathbf{0}$ \\
4 & 11 & 0 & 0 & 0 & 2 & $\mathbf{0}$ & $\mathbf{0}$ & $\mathbf{0}$ \\
5 & 34 & 2 & 0 & 0 & 4 & $\mathbf{0}$ & $\mathbf{0}$ & $\mathbf{2}$ \\
6 & 156 & 10 & 0 & 4 & 16 & $\mathbf{0}$ & $\mathbf{0}$ & $\mathbf{8}$ \\
7 & 1044 & 110 & 40 & 130 & 102 & $\mathbf{2 0}$ & $\mathbf{2 4}$ & $\mathbf{4 8}$ \\
8 & 12346 & 1722 & 1166 & 1767 & 1201 & $\mathbf{5 6 5}$ & $\mathbf{6 8 8}$ & $\mathbf{5 1 1}$ \\
9 & 274668 & $51039^{*}$ & 43811 & 42595 & 19001 & $\mathbf{2 1 6 4 2}$ & $\mathbf{2 5 7 7 4}$ & $\mathbf{8 7 4 3}$ \\
10 & 12005168 & $2560606^{*}$ & 2418152 & 1412438 & $645146^{* *}$ & $\mathbf{1 2 1 4 8 5 1}$ & $\mathbf{1 4 1 6 5 2 7}$ & $\mathbf{3 2 8 6 8 3}$ \\
\hline
\end{tabular}

Table 2: Fraction of non-isomorphic graphs with cospectral mates, until $n=10$, w.r.t. to the matrices $A, A \& \neg A, L, Q, M_{\ell} s, \hat{M}$ and $Q_{\hat{M}}$.

\begin{tabular}{|l|l|l|l|l|l||l|l|l|}
\hline \hline$n$ & \# graphs & $A$ & $A \& \neg A$ & $L$ & $Q$ & $\mathbf{M}_{\ell} \mathrm{s}$ & $\hat{\mathbf{M}}$ & $Q_{\hat{\mathbf{M}}}$ \\
\hline 2 & 2 & 0 & 0 & 0 & 0 & $\mathbf{0}$ & $\mathbf{0}$ & $\mathbf{0}$ \\
3 & 4 & 0 & 0 & 0 & 0 & $\mathbf{0}$ & $\mathbf{0}$ & $\mathbf{0}$ \\
4 & 11 & 0 & 0 & 0 & 0.182 & $\mathbf{0}$ & $\mathbf{0}$ & $\mathbf{0}$ \\
5 & 34 & 0.059 & 0 & 0 & 0.118 & $\mathbf{0}$ & $\mathbf{0}$ & $\mathbf{0 . 0 5 9}$ \\
6 & 156 & 0.064 & 0 & 0.026 & 0.103 & $\mathbf{0}$ & $\mathbf{0}$ & $\mathbf{0 . 0 5 1}$ \\
7 & 1044 & 0.105 & 0.038 & 0.125 & 0.098 & $\mathbf{0 . 0 1 9}$ & $\mathbf{0 . 0 2 3}$ & $\mathbf{0 . 0 4 6}$ \\
8 & 12346 & 0.139 & 0.094 & 0.143 & 0.097 & $\mathbf{0 . 0 4 6}$ & $\mathbf{0 . 0 5 6}$ & $\mathbf{0 . 0 4 1}$ \\
9 & 274668 & 0.186 & 0.160 & 0.155 & 0.069 & $\mathbf{0 . 0 7 9}$ & $\mathbf{0 . 0 9 4}$ & $\mathbf{0 . 0 3 2}$ \\
10 & 12005168 & 0.213 & 0.201 & 0.118 & 0.054 & $\mathbf{0 . 1 0 1}$ & $\mathbf{0 . 1 1 8}$ & $\mathbf{0 . 0 2 7}$ \\
\hline
\end{tabular}

One important fact is that $Q_{\hat{M}}$ is more efficient than $Q$, since the fraction of cospectral mates reduces by a factor of 2 or more. As in the case of $Q$, the fraction of cospectral mates, for $Q_{\hat{M}}$, decreases monotonically with the number of vertices. We also note that $\hat{M}$ and the set of $M_{\ell}$ s are more efficient than the other classical matrices. 
The conjecture of Haemers and Spence [8], that the fraction of graphs with cospectral mates, with respect to $A, A \& \neg A$ and $Q$ matrices, tends to zero as $n$ goes to infinity, seems to be further valid for $Q_{\hat{M}}$.

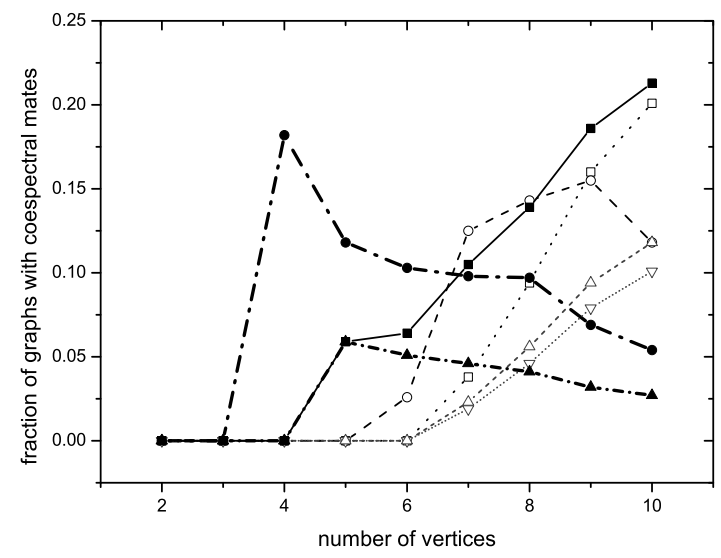

Figure 1: The fraction of cospectral mates w.r.t. to the matrices versus number of vertices $n: A$ (black solid line - square), A\& $\neg A$ (black dashed line - blank square), L (black dotted line - blank circle), $Q$ (black dot-dashed line - circle), $\hat{M}$ (dark gray short dotted line blank up triangle), $M_{\ell}$ s (dark gray short dashed line - blank down triangle), $Q_{\hat{M}}$ (dark gray short dot-dashed line - up triangle).

Figure 1 presents the fraction of cospectral mates versus the order of the graphs in Table 2, providing a comparative picture that makes clear that the fraction of graphs with cospectral mates vary differently according to the matrix sets. Observe the superiority of $Q_{\hat{M}}$ in differentiating graphs.

Therefore, our analysis of neighborhood based matrices clearly reveals that the neighborhood structure is a relevant feature in the study of graph spectra. According to Wilson and Zhu [10], combining spectra of diverse matrices associated to graphs may be a good approach to better differentiate graphs. In order to investigate the impact of these combinations, we set up the Table 3 which summarizes our results.

Observe that, for $n<10$, there is no cospectral mate between $\hat{M}$ and $Q_{\hat{M}}$, and, for $n=10$, the fraction of cospectral mates reduces to 0.00064 .

Comparing the results presented in the last column of Table 3 with the results concerning the method proposed by Godsil and MaKay [7], the GM* switching method [8], we conclude that they are equivalent for $n<10$, reducing the cospectral incidence to zero. However, for $n=10$, the combined spectra leads to a slightly better result: $\hat{M} \& Q_{\hat{M}}$ (7712 common cospectral mates) and the lower bound of GM* (9480 cospectral mates). 
Table 3: Number of non-isomorphic graphs with cospectral mates w.r.t. $Q, \hat{M}, Q_{\hat{M}}$ and the combinations $Q_{\hat{M}} \& Q, \hat{M} \& Q$, and $\hat{M} \& Q_{\hat{M}}$.

\begin{tabular}{|l|l|l|l|l||l|l|l|}
\hline \hline$n$ & \# graphs & $Q$ & $\hat{M}$ & $Q_{\hat{\mathbf{M}}}$ & $Q_{\hat{\mathbf{M}}} \& Q$ & $\hat{\mathbf{M}} \& Q$ & $\hat{\mathbf{M}} \& Q_{\hat{\mathbf{M}}}$ \\
\hline 2 & 2 & 0 & 0 & 0 & $\mathbf{0}$ & $\mathbf{0}$ & $\mathbf{0}$ \\
3 & 4 & 0 & 0 & 0 & $\mathbf{0}$ & $\mathbf{0}$ & $\mathbf{0}$ \\
4 & 11 & 0 & 0 & 2 & $\mathbf{0}$ & $\mathbf{0}$ & $\mathbf{0}$ \\
5 & 34 & 4 & 0 & 8 & $\mathbf{0}$ & $\mathbf{0}$ & $\mathbf{0}$ \\
6 & 156 & 16 & 0 & 8 & $\mathbf{0}$ & $\mathbf{0}$ & $\mathbf{0}$ \\
7 & 1044 & 102 & 24 & 48 & $\mathbf{4 8}$ & $\mathbf{0}$ & $\mathbf{0}$ \\
8 & 12346 & 1201 & 688 & 511 & $\mathbf{5 0 3}$ & $\mathbf{0}$ & $\mathbf{0}$ \\
9 & 274668 & 19001 & 25774 & 8743 & $\mathbf{8 5 2 4}$ & $\mathbf{4}$ & $\mathbf{0}$ \\
10 & 12005168 & 645146 & 1416510 & 328683 & $\mathbf{1 8 3 4 4}$ & $\mathbf{7 7 6 6}$ & $\mathbf{7 7 1 2}$ \\
\hline
\end{tabular}

\section{Discussion and Concluding Remarks}

In the present work, we analysed graph cospectrality, for $n<11$, with respect to the matrices: $M_{\ell} \mathrm{s}, \hat{M}$, and $Q_{\hat{M}}$. Our main results, summarized in the last columns of Tables 1 and 3 (boldface type), reveal the superiority of our approach to distinguish the graphs, concerning to the cospectrality, in comparison to other previously reported matrices $Q$ and $A$.

The signless laplacian of neighborhood matrix $Q_{\hat{M}}$ offers lower cospectrality incidences than those provided by $Q$, with a significant reduction factor $\sim 2$, mainly because both of them are decrescent with the number of vertices $n$, at least, until $n=10$. It seems that Haemers and Spence conjecture works for $Q$ and $Q_{\hat{M}}$ matrices: the fraction of graphs with a cospectral mate tends to zero as $n \rightarrow \infty$.

Following Wilson and Zhu [10], we combined different spectra to reduce the cospectrality incidence among graphs. The results reveal that this approach can drastically reduce the cospectrality among graphs for the combinations $\hat{M} \& Q_{\hat{M}}$ and $\hat{M} \& Q$, confirming the efficiency of neighborhood matrices approach for the cospectrality problem. Finally we observe that the method of combining spectra is slightly better than the GM* method presented in [8] at least for $n=10$ (they are equivalent for $n<10$ ).

\section{Acknowledgements}

The authors thank CNPq and FAPESB (Brazilian agencies) and INCT-SC for partial support. The first author is also very thankful for fruitful discussions with W. H. Haemers.

\section{References}

[1] R. F. S. Andrade, J. G. V. Miranda, and T. P. Lobão. Neighborhood Properties of Complex Networks, Phys. Rev. E 73: 046101, 2006. 
[2] R. F. S. Andrade, J. G. V. Miranda, S. T. R. Pinho, and T. P. Lobao. Characterization of complex networks by higher order neighborhood properties. Eur. Phys. J. B 61: 247-256 (2008).

[3] A. E. Brouwer and E. Spence, Cospectral Graphs on 12 Vertices. The Eletronics J. Combin. 16: 1-3, 2009.

[4] F. Buckley and F. Harary. Distance in Graphs. Addison-Wesley Publishing Company, New York, 1990.

[5] D. M. Cvetkovic; M. Doob, and H. Sachs. M. Cvetkovi\&cacute, Spectra of Graphs: Theory and Applications. Academic Press, New York, 1997.

[6] D. M. Cvetkovic; P. Rowlinson, and S. K. Simić. Eingenvalue bounds for the signless Laplacian. Publications de L'Institut Mathematique 85: 11-27 (2007).

[7] C. D. Godsil, B. D. McKay. Constructing cospetral graphs. Aequations Math. 25: 257-268, 1982.

[8] W. H. Haemers and E. Spence. Enumeration of Cospectral Graphs. Eur. J. Combin. 25, 199-211, 2004.

[9] B. McKay. Combinatorial Data. http://cs.anu.edu.au/ bdm/data/graphs.html.

[10] P. Zhu and R.C. Wilson. A Study of Graph Spectra for Comparing Graphs. Technical Report, York University, UK, 2005. 